\title{
Recursive Noise Reduction for Digital Radiography Images
}

\author{
Vladimir S. Ostojić, Đorđe S. Starčević, and Vladimir S. Petrović
}

\begin{abstract}
Modern digital radiography images are large, suggesting that a denoising algorithm should be computationally efficient. In this paper, we propose a homomorphic recursive noise reduction approach. The proposed method was compared to anisotropic diffusion on a database consisting of 47 clinical radiography images, and was subjectively deemed better at noise reduction. The proposed approach was shown to require $50 \%$ fewer iterations to produce subjectively the same results. Objective comparison of the algorithms through a structural similarity index and peak signal-to-noise ratio showed that the proposed method outperforms anisotropic diffusion for various signal-to-noise ratios.
\end{abstract}

Index Terms - digital radiography, homomorphic filtering, image noise reduction, recursive filtering.

\section{INTRODUCTION}

U SING low radiation doses for radiography imaging is beneficial both for the patient and the medical staff, and is common when sequences of images are acquired, like in angiography [1]. Radiation dose reduction is possible with modern high dynamic range detectors that allow a wide range of exposure levels while still producing good contrast images [2]. A negative effect of the radiation reduction strategy is the involuntary increase of the quantum noise level which manifests through a negative effect on image quality. A high quantum noise level associated with low dose imaging produces the quantum mottle artefact that has a distracting effect on the diagnostician as subtle details can be overlooked [3]. In addition, modern contrast and detail enhancement algorithms tend to increase the impact of the noise on the

Paper received April 13, 2018; accepted April 25, 2018. Date of publication July 31, 2018. The associate editor coordinating the review of this manuscript and approving it for publication was Prof. Branimir Reljin.

This paper is a revised and expanded version of the paper presented at the 25th Telecommunications Forum TELFOR 2017 [18].

This research is supported by grants TR32035and TR32040 of Serbian Ministry of Science.

Vladimir S. Ostojić is with the University of Novi Sad, Serbia Faculty of Technical Sciences, Department of Power, Electronics and Telecommunications (e-mail: vladimir.ostojic@uns.ac.rs).

Đorđe S. Starčević is with the University of Novi Sad, Serbia, Faculty of Technical Sciences, Department of Power, Electronics and Telecommunications (e-mail: djordje.starcevic@uns.ac.rs).

Vladimir S. Petrović is with the University of Novi Sad, Serbia, Faculty of Technical Sciences, Department of Power, Electronics and Telecommunications (e-mail:vladimir.petrovic@uns.ac.rs). image quality [2]. As can be deduced from the previous statements, noise should be reduced in radiography images to achieve a better image quality, and this should be done as a pre-processing step relative to image enhancement.

There are various and numerous approaches to radiography image denoising. It should be noted that fine anatomical details should be preserved in the process of noise reduction, as they may carry valuable diagnostic information. Hence, simple indiscriminate methods, like the well-known Gaussian or Wiener filtering [4], are not suitable for radiography images. Several approaches use correlation between anatomical structures over multiple scales with the purpose of distinguishing them from noise. An algorithm that uses Laplacian pyramid [5] decomposition for this purpose was proposed in [1], while approaches that use wavelet decomposition were proposed in [6] and [7]. A simplified variation of these approaches, that uses only the intensity of the coefficients obtained in the multi-scale image decomposition instead of their correlation, was used as a denoising step of medical image enhancement algorithm in [8]. Image decomposition to low-frequency and high-frequency components, and subsequent high-frequency component value manipulation was proposed in [2]. An approach that uses similarity between neighborhood of a voxel to evaluate its contribution in the denoising process of volumetric images was proposed in [9]. Adaptation of the previous algorithm with the task of noise removal in $2 \mathrm{D}$ radiography imaging was proposed in [10].

Non-linear approaches for image smoothing and denoising such as the bilateral filter [11] and non-local means filtering [12] are impractical for radiography images because of their high complexity and size of the images (ranging from 9 megapixels for diagnostic x-ray [13] up to 25 megapixels for digital mammography [14]). Recently, edge preserving low complexity recursive algorithms that employ recursive filtering have been proposed. A recursive version of the bilateral filter and a gradient domain bilateral filter were proposed in [15]. A bi-exponential recursive approach for image smoothing was proposed in [16]. A recursive anisotropic diffusion denoising algorithm was proposed in [17]. In this paper, we propose a homomorphic recursive anisotropic diffusion algorithm for radiography image denoising. Initial research and analysis of this idea was presented in [18].

Section II outlines the anisotropic diffusion [19] as the basis for the recursive anisotropic diffusion. Recursive anisotropic diffusion is explained in Section III. Homomorphic adaptation of recursive denoising for radiography images is explained in section IV. Evaluation and the results are presented in Section V. Section VI concludes the paper. 


\section{ANISOTROPIC DIFFUSION}

Anisotropic diffusion (AD) [19] is an iterative denoising strategy that updates the pixel value based on the intensity difference with its nearest neighbors. $\mathrm{AD}$ is defined through a partial differential equation:

$$
\frac{\partial I}{\partial t}=\operatorname{div}(c(x, y, t) \nabla I)
$$

where $I$ represents the image, $x$ and $y$ denote spatial coordinates, $t$ represents the time, $\operatorname{div}(\cdot)$ stands for divergence while $\nabla(\cdot)$ designates the gradient operator. A discrete version of the equation given in (1) is:

$$
\begin{gathered}
I_{t+1}(x, y)=I_{t}(x, y)+\lambda c_{n} d_{n}+\lambda c_{s} d_{s}+\lambda c_{e} d_{e} \\
+\lambda c_{w} d_{w}
\end{gathered}
$$

where $t$ denotes the iteration, $x$ and $y$ are the discrete spatial coordinates and $\lambda$ is a constant from the range $[0,0.25]$. Coefficients $d_{i}, i \epsilon\{n, s, e, w\}$ are the differences between the value of pixel at coordinates $(x, y)$ and values of its nearest neighbors, with a coordinate origin positioned at the top left corner of the image:

$$
\begin{aligned}
& d_{n}=I_{t}(x-1, y)-I_{t}(x, y) \\
& d_{s}=I_{t}(x+1, y)-I_{t}(x, y) \\
& d_{e}=I_{t}(x, y+1)-I_{t}(x, y) \\
& d_{w}=I_{t}(x, y-1)-I_{t}(x, y)
\end{aligned}
$$

Conduction coefficients $c_{i}, i \in\{n, s, e, w\}$ correspond to differences $d_{i}$ :

$$
c_{i}=e^{-\left(\frac{d_{i}}{k}\right)^{2}}
$$

where $k$ is a constant used to control the influence of the differences $d_{i}$ to the coefficients $c_{i}$, and may also be considered as a way to control edge sensitivity and is usually established empirically. Dependence of coefficients $c_{i}$ on the value of constant $k$ is shown in Fig. 1.

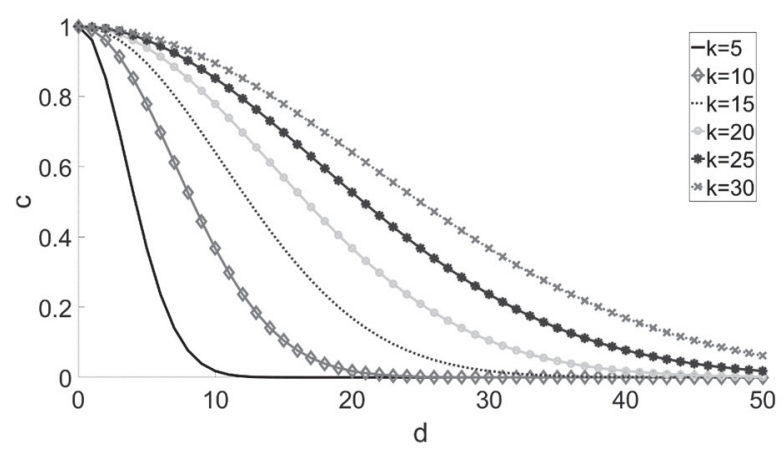

Fig. 1. Dependence of conduction coefficient $c_{i}$ on the value of constant $k$.

As can be deduced from (2) and (4), the value of the central pixel $I_{t}(x, y)$ is nonlinearly updated in each iteration. If difference $d_{i}$ has a high value, the corresponding coefficient $c_{i}$ will be small, meaning that neighbors that have values more similar to that of the central pixel contribute more to its value change. In practice, this results in the removal of small intensity fluctuations in homogenous image regions, thus effectively reducing noise. On the other hand, neighbors that have a significantly different value compared to the value of the central pixel (e.g. on edges) do not contribute significantly to its value change, which leads to image edge preservation.

\section{RECURSIVE ANISOTROPIC DIFFUSION}

In the first iteration of $\mathrm{AD}$ only four nearest neighbors are used, while the neighbors of the nearest neighbors are implicitly used in the second iteration, effectively increasing filter support. The support will keep increasing with the increase of iteration count. In contrast, recursive filters have infinite support by design. This inspires the combining of the recursive filtering with the nonlinear edge preservation qualities of the $\mathrm{AD}$, leading to recursive anisotropic diffusion (RAD) [17].

Let us analyze the influence of only one neighbor on the central pixel value change:

$$
I_{t+1}(x, y)=I_{t}(x, y)+\lambda c_{n} d_{n}
$$

By taking the expression given in (3) and placing it in (5) we get:

$$
I_{t+1}(x, y)=\left(1-\lambda c_{n}\right) I_{t}(x, y)+\lambda c_{n} I_{t}(x-1, y)
$$

Replacing $I_{t}(x-1, y)$ with $I_{t+1}(x-1, y)$ (iteration index has changed) provides recursive filtering:

$$
I_{t+1}(x, y)=\left(1-\lambda c_{n}\right) I_{t}(x, y)+\lambda c_{n} I_{t+1}(x-1, y)
$$

This manipulation ensures that all pixels that have a smaller value $x$ coordinate than pixel $I_{t+1}(x, y)$ contribute in its update in the iteration. This is important from the support standpoint, as filter support does not iteratively expand but is infinitely large by design.

This principle is applicable to each neighbor of the central pixel, resulting in neighbor recursion image definition (analogously to (5)) $J_{t+1}^{i}, i \in\{n, s, e, w\}$ :

$$
J_{t+1}^{i}(x, y)=I_{t}(x, y)+\lambda C_{i} D_{i}
$$

where $t$ is the iteration, $\lambda$ is the same as in (2).Coefficients $D_{i}, i \in\{n, s, e, w\}$ correspond to the intensity difference between the central pixel $I_{t-1}(x, y)$ and the neighboring pixel in the recursive neighbor image:

$$
\begin{aligned}
D_{n} & =J_{t}^{n}(x-1, y)-I_{t-1}(x, y) \\
D_{s} & =J_{t}^{s}(x+1, y)-I_{t-1}(x, y) \\
D_{e} & =J_{t}^{e}(x, y+1)-I_{t-1}(x, y) \\
D_{w} & =J_{t}^{w}(x, y-1)-I_{t-1}(x, y)
\end{aligned}
$$

Coefficients $C_{i}$ are defined analogously to $c_{i}$ :

$$
C_{i}=e^{-\left(\frac{D_{i}}{k}\right)^{2}}
$$

Upon analyzing (8) and (9), it becomes clear that images $J_{t}^{i}, i \in\{n, s, e, w\}$ are calculated recursively, e.g. $J_{t}^{n}$ is 
obtained by filtering in the direction of the increasing $x$, while $J_{t}^{e}$ is obtained by filtering in the decreasing $y$ direction, etc.

$\mathrm{RAD}$ is defined analogously to AD:

$$
\begin{gathered}
I_{t}(x, y)=I_{t-1}(x, y)+\lambda C_{n} D_{n}+\lambda C_{s} D_{s} \\
+\lambda C_{e} D_{e}+\lambda C_{w} D_{w}
\end{gathered}
$$

It was shown that RAD outperforms $\mathrm{AD}$ in denoising nonmedical images and that it can be used to obtain superior results with fewer iterations [17]. These conclusions make RAD an interesting algorithm from the standpoint of denoising radiographic images as they are large with respect to pixel count, emphasizing the need for the number of potentially used iterations to be low.

\section{ADAPTATION OF RAD FOR RADIOGRAPHY IMAGES}

Radiography image noise is predominantly of quantum type, since it is associated with a random distribution of the x-ray photons registered by the digital detector [2]. Let us denote the average number of photons that the source emits per second in the direction of the digital image detector with $\rho$. The probability of detecting $X$ photons during a period of $T$ seconds is defined by the Poisson distribution [20]:

$$
P(X \mid \rho T)=\frac{e^{-\rho T}(\rho T)^{X}}{X !}
$$

Expected photon count $\mu$ is equal to the variance of $X$ :

$$
E\{X \mid \rho T\}=\rho T=\mu=\operatorname{var}\{X \mid \rho T\}
$$

The Poisson noise can be defined as [21]:

$$
n=X-\mu
$$

The Poisson noise thus has a zero mean and the variance:

$$
\operatorname{var}\{n \mid \rho T\}=\operatorname{var}\{X \mid \rho T\}=\mu
$$

It should be noted that, since the noise variance depends on the expected value of $X$, it is signal dependent.

The observed number of photons $X$ is converted into pixel intensities as [20]:

$$
I=b+g \cdot X
$$

where $b$ represents the bias and $g$ is the detector gain. Bias $b$ is used in the detector calibration process to ensure that $I=0$ if there is no radiation. Under the assumption that detector is properly calibrated, $b$ can be omitted from (16). Inserting (14) into (16) gives:

$$
I=g \cdot X=g \cdot \mu+g \cdot n=\hat{I}+\hat{n}
$$

where $\hat{I}=g \cdot \mu$ and $\hat{n}=g \cdot n$. Noise $\hat{n}$ still has a zero mean, but is no longer Poisson as its variance is:

$$
\operatorname{var}\{\hat{n}\}=g^{2} \mu=g \hat{I} \neq \hat{I}
$$

Starting with (17) we get:

$$
I=\hat{I}+\hat{n}=\hat{I}+\sqrt{\hat{I}} \frac{\hat{n}}{\sqrt{\hat{I}}}=\hat{I}+\sqrt{\hat{I}} \cdot m
$$

where $m$ stands for noise defined as:

$$
m=\frac{\widehat{n}}{\sqrt{\hat{I}}}=\frac{\widehat{n}}{\sqrt{g \cdot \mu}}=\frac{\sqrt{g} \cdot \widehat{n}}{g \sqrt{\mu}}=\sqrt{g} \cdot \check{n}
$$

and $\check{n}$ is the noise $\hat{n}$ normalized to unitary variance. It is important to note that $m$ is independent of signal intensity. Under the assumption that the variance of $m$ is small (it was shown that $g$ has small values in practice [20]), taking the square root of (19) and using the Maclaurin series gives:

$$
\sqrt{I} \approx \sqrt{\hat{I}}+\frac{1}{2} m
$$

We conclude that taking the square root of the pixel intensity will produce a signal with noise that is approximately independent of the pixel intensity. As RAD is designed for additive Gaussian noise removal, the square root should be used prior to denoising. Values obtained after denoising should be squared to ensure return to the original image value domain. Thus, the proposed approach is homomorphic.

\section{EVAluAtion AND RESUlts}

To evaluate the proposed approach, we have used a database that consists of 47 digital radiography images. The images were acquired using Varian PaxScan4343 flat panel detector, and the acquisition took place during regular clinical routines. All images have the same size of 3072 x 3072 pixels. Detector pixels are square with a length of $139 \mu \mathrm{m}$.

Noise reduction is only one step in the radiography image processing pipeline [22], thus the effect of denoising should be evaluated through the analysis of the images that were processed completely, i.e. they are in the form that will be presented to the radiologist. Therefore, we employed the proposed noise reduction algorithm as a pre-processing step, bearing in mind that detail and contrast enhancements increase noise prominence [2]. The algorithm proposed in [22] was used to perform the image enhancement. This algorithm, based on multiscale detail and contrast enhancement, includes dynamic value range mapping and adjustment of the image tone scale.

Choice of parameters for the noise reduction algorithm was made based on AD performance, in such a way that would ensure efficient noise removal while preserving image edges on five randomly selected images from the database. It should be noted that the parameters were not optimized for a specific type of the imaged anatomy, but were set to the same value for each image in the entire database. The values chosen were $\lambda=0.25$ and $k=30$. Each image in the database was processed with AD and RAD, and the results were compared visually. An example of an image after denoising is shown in Fig. 2.Images in Fig. 2 show that RAD produces satisfactory results with 
only two iterations (Fig. 2 (f)), while AD requires four iterations to produce subjectively the same noise reduction results (Fig. 2 (i)).

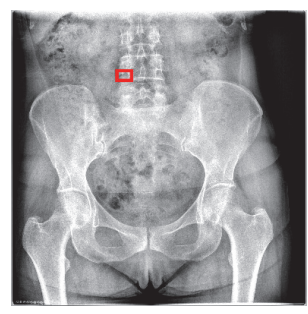

(a)

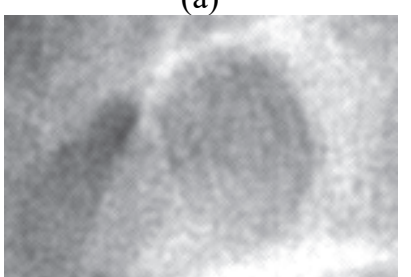

(c)

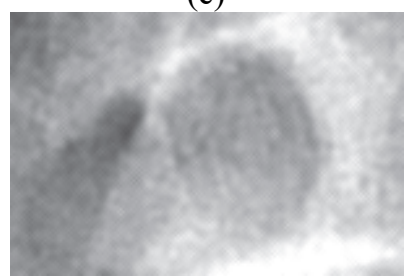

(e)

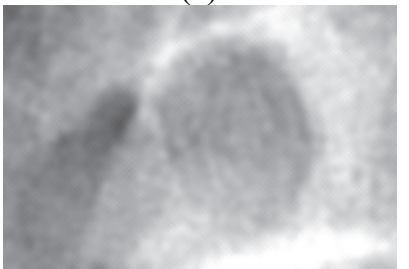

$(\mathrm{g})$

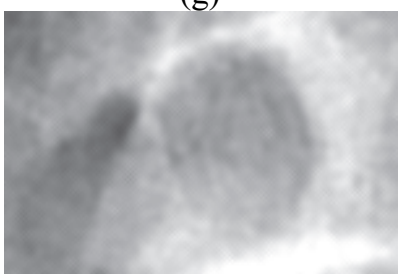

(i)

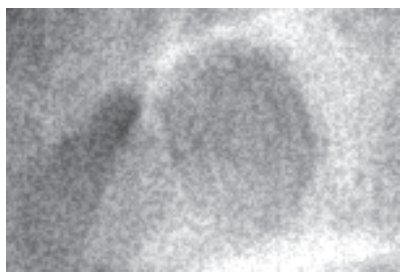

(b)

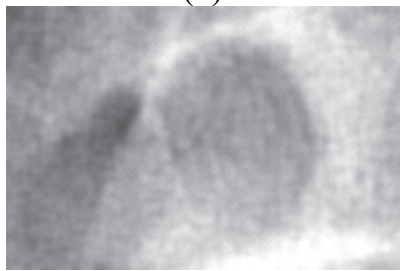

(d)

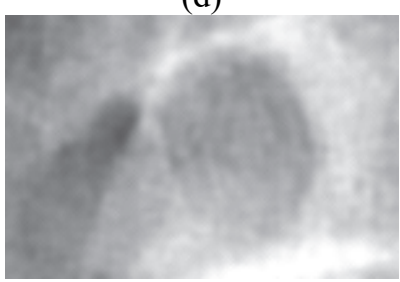

(f)

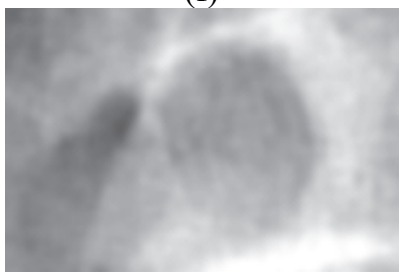

(h)

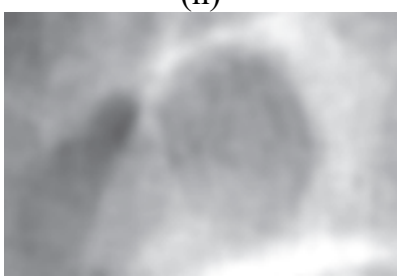

(j)
Fig. 2. Comparison of AD and RAD. (a) Pelvis image with marked segment shown in (b). Result of AD with one (c), two (e), three (g) and four (i) iterations. Result of RAD

with one (d), two (f), three (h) and four (j) iterations.

Figs. 3, 4, 5 and 6 serve for further subjective comparison of performance of $\mathrm{AD}$ (four iterations) and RAD (two iterations) on radiography images showing different anatomies. Equivalent denoising performance was achieved on all other images in the database.

Objective comparison of noise reduction algorithms on clinical images is not straightforward due to the fact that the clinical images already contain noise, hence there are no noise-free reference images. Therefore, we compared images before enhancement, in the homomorphic domain, to ensure that the noise is independent of the pixel values (as previously described in Section IV). Images processed with boxcar low-pass $5 \times 5$ filter were established as the reference noise-free images [23]. Gaussian noise of several different variances was added to these images. The

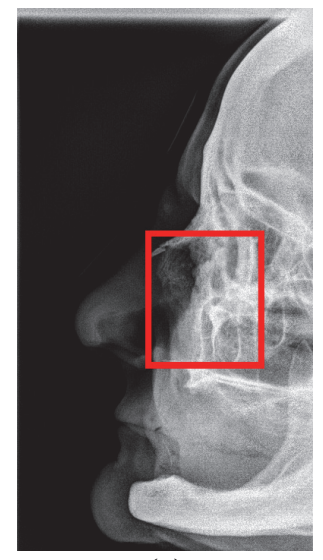

(a)

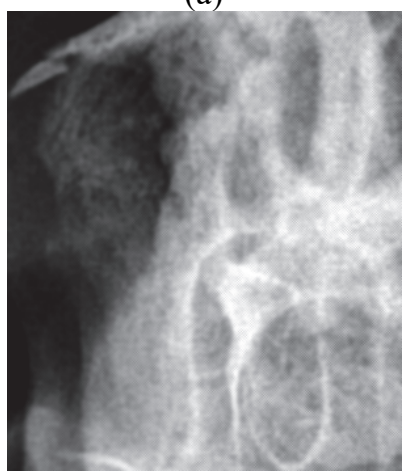

(c)

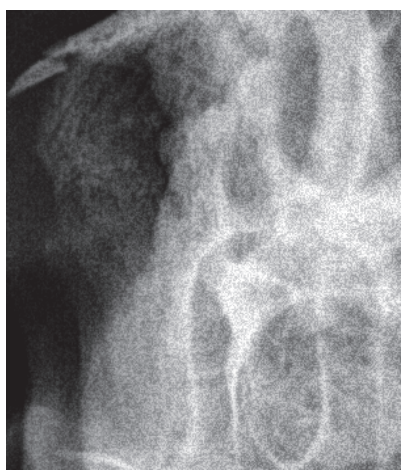

(b)

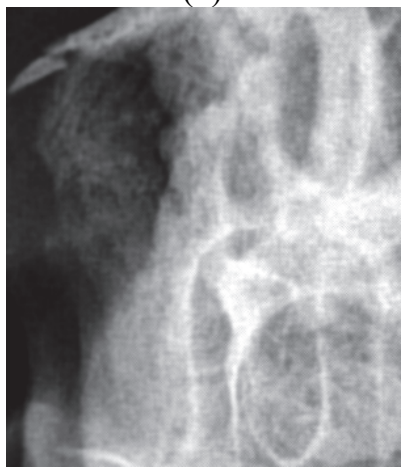

(d)
Fig. 3. Comparison of AD and RAD on a facial bones radiography image. (a) Original image with marked example region, (b) zoomed region with noise, (c) region after processing with $\mathrm{AD}$, (d) region after processing with RAD.

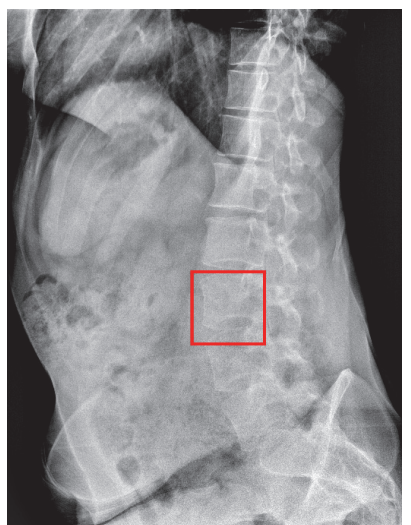

(a)

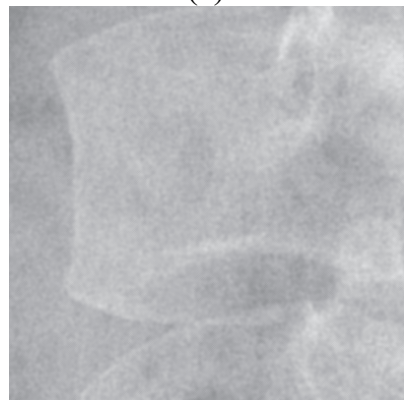

(c)

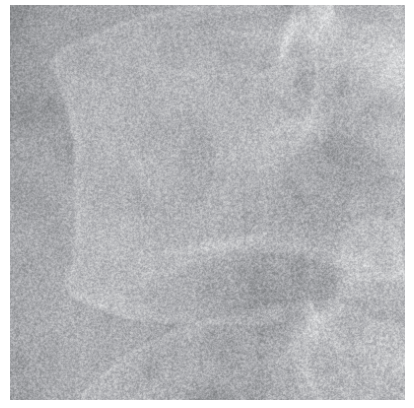

(b)

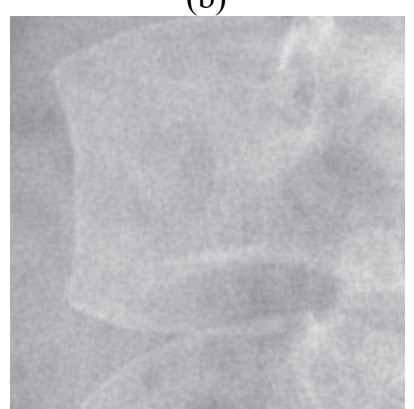

(d)
Fig. 4. Comparison of $\mathrm{AD}$ and $\mathrm{RAD}$ on a lumbar spine radiography image. (a) Original image with marked example region, (b) zoomed region with noise, (c) region after processing with $\mathrm{AD}$, (d) region after processing with RAD. 


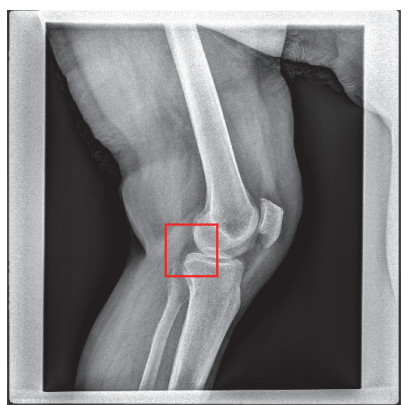

(a)

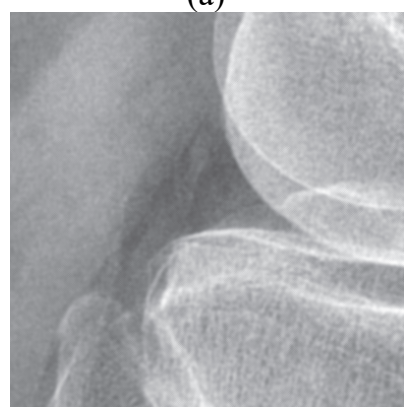

(c)

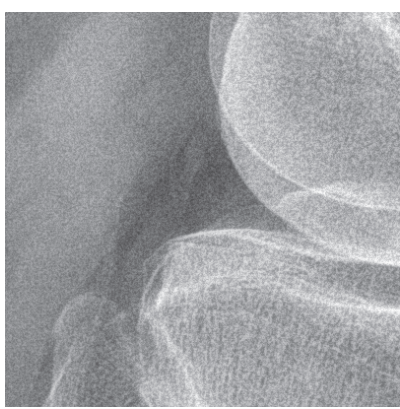

(b)

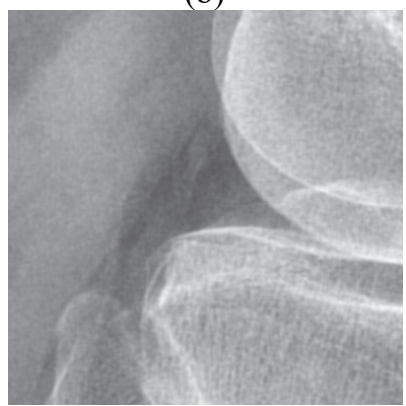

(d)
Fig. 5. Comparison of $\mathrm{AD}$ and $\mathrm{RAD}$ on a knee image.

(a) Original image with marked example region, (b) zoomed region with noise, (c) region after processing with $\mathrm{AD}$, (d) region after processing with RAD.

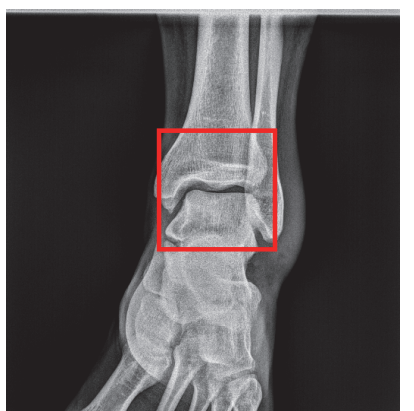

(a)

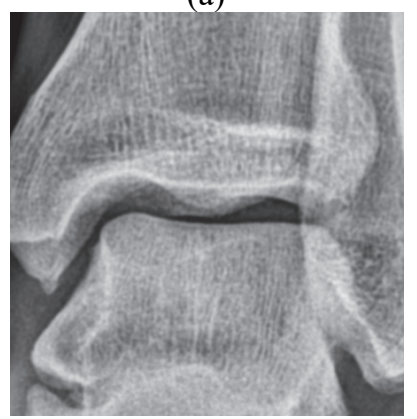

(c)

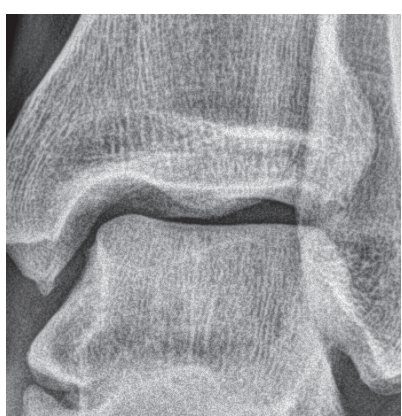

(b)

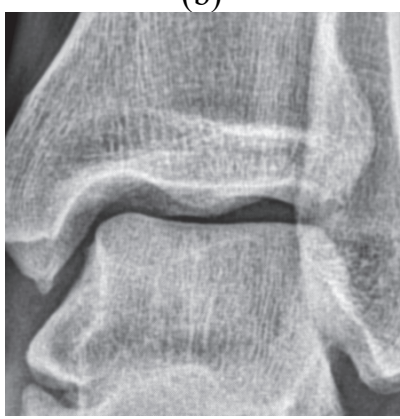

(d)
Fig. 6. Comparison of AD and RAD on an ankle image.

(a) Original image with marked example region, (b) zoomed region with noise, (c) region after processing with $\mathrm{AD}$, (d) region after processing with RAD.

denoising performances of $\mathrm{AD}$ and RAD were evaluated through comparison of the mean values, on the entire database, of structural similarity index (SSIM) [24] as well as well-known peak signal-to-noise ratio (PSNR) with reference to the noise-free images.

SSIM for two images $A$ and $B$ of common size $P x Q$ is defined as:

$$
\begin{aligned}
& \operatorname{SSIM}(A, B) \\
& =\frac{1}{P Q} \sum_{x, y} \frac{\left(2 \mu_{A} \mu_{B}+c_{1}\right)\left(2 \sigma_{A B}+c_{2}\right)}{\left(\mu_{A}^{2}+\mu_{B}^{2}+c_{1}\right)\left(\sigma_{A}^{2}+\sigma_{B}^{2}+c_{2}\right)}
\end{aligned}
$$

where $\mu_{A}$ and $\mu_{B}$ are the average values and $\sigma_{A}^{2}$ and $\sigma_{B}^{2}$ are variances within $\mathrm{A}$ and $\mathrm{B}, \sigma_{A B}$ is the covariance of $\mathrm{A}$ and $\mathrm{B}$, while $c_{1}$ and $c_{2}$ are stabilization constants which depend on the dynamic pixel value range. The SSIM results are obtained on the entire evaluation database. For fair comparison, we used $\lambda=0.25, k=30$ and two iterations for both algorithms. Fig. 7 clearly shows that $\mathrm{RAD}$ outperforms $\mathrm{AD}$ in radiography image denoising.

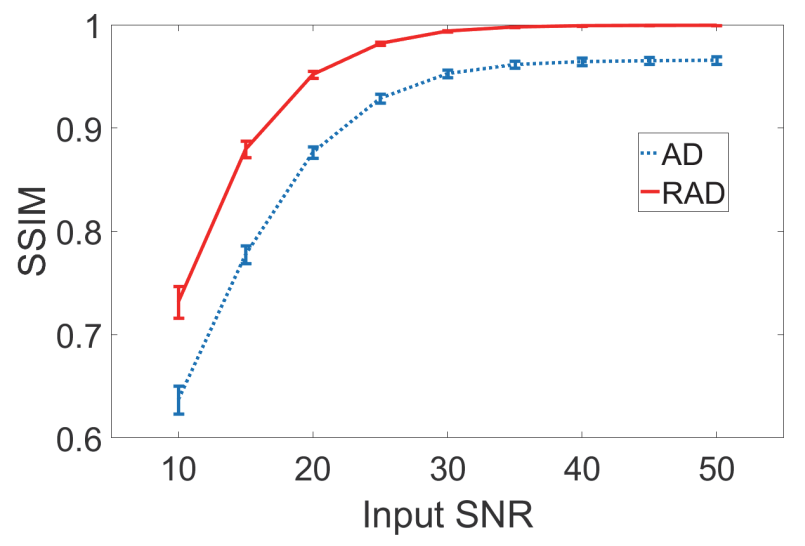

Fig. 7. Comparison of $\mathrm{AD}$ and $\mathrm{RAD}$ through mean SSIM obtained on the database.

PSNR is defined as:

$$
P S N R=10 \log \frac{M^{2}}{\frac{1}{N} \sum_{x} \sum_{y}(I(x, y)-\hat{I}(x, y))^{2}}
$$

where $M$ represents the maximal pixel value, $N$ is the image pixel count, $I$ is the original image whereas $\hat{I}$ is the image after denoising. Similarly as with SSIM, the results are obtained on the entire evaluation database, parameters are set to $\lambda=0.25, k=30$ with two iterations for both algorithms. Comparison of obtained PSNR values is presented in Fig. 8, depicting the objective advantage of $\mathrm{RAD}$ over $\mathrm{AD}$ in image noise reduction.

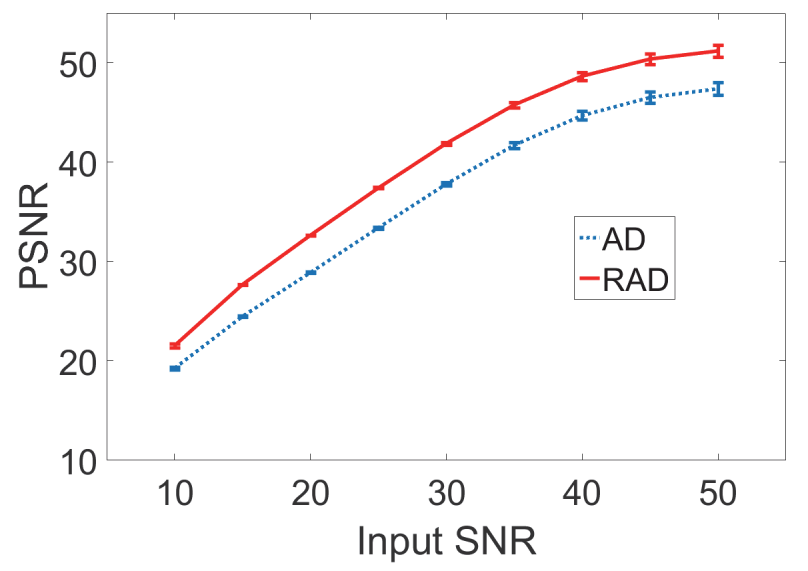

Fig. 8. Comparison of AD and RAD through mean PSNR obtained on the database. 


\section{CONCLUSION}

In this paper, we proposed a homomorphic recursive anisotropic diffusion approach to noise reduction in digital radiography images. The proposed approach utilizes recursive filters, which have infinite support by design, to achieve equivalent noise reduction in fewer iterations than conventional anisotropic diffusion. Homomorphism was employed with the purpose of reducing noise that is approximately independent of the pixel intensity.

The recursive denoising method was evaluated within a radiography image processing pipeline, more precisely as its pre-processing step. It was shown that the proposed approach produces a visually satisfactory result with only two iterations compared to AD that needs twice as many. Objective comparison on a database of 47 clinical images was performed through comparison of SSIM and PSNR values and showed that homomorphic RAD outperforms homomorphic AD.

\section{REFERENCES}

[1] M. Hensel, U. Brummund, T. Pralow, R. R. Grigat, "Noise Reduction with Edge Preservation by Multiscale Analysis of Medical X-Ray Image Sequences," Bildverarbeitungfür die Medizin 2005: Algorithmen - Systeme - Anwendungen Proceedings des Workshops vom 13. - 15. März 2005 in Heidelberg, H. P. Meinzer, H. Handels, A. Horsch, T. Tolxdorff, Ed. Berlin: Springer Berlin Heidelberg, 2005, pp. 55-59.

[2] M. Couwenhoven, W. Sehnert, X. Wang, M. Dupin, J. Wandtke, S. Don, R. Kraus, N. Paul, N. Halin, and R. Sarno "Observer study of a noise suppression algorithm for computed radiography images," Proc. SPIE, Medical Imaging 2005: Image Perception, Observer Performance, and Technology Assessment, vol. 5749, pp. 318-327, 2005.

[3] S. L. Solomon, R. G. Jost, H. S. Glazer, S. S. Sagel, D. J. Anderson, and P. L. Molina, "Artifacts in computed radiography," American Journal of Roentgenology, vol. 157, no. 1, pp. 181-185, 1991.

[4] R. C. Gonzalez and R. E.Woods, Digital Image Processing (3rd Edition). Upper Saddle River, NJ, USA: Prentice-Hall, Inc., 2006

[5] P. Burt, and E. Adelson, "The Laplacian Pyramid as a Compact Image Code", IEEE Trans. Commun., vol. Com-31, no. 4, 1983.

[6] A. Pizurica, W. Philips, I. Lemahieu, and M. Acheroy, "A versatile wavelet domain noise filtration technique for medical imaging," IEEE Trans. Med. Imag., vol. 22, no. 3, pp. 323-331, March 2003.

[7] P. Bao and L. Zhang, "Noise reduction for magnetic resonance images via adaptive multiscale products thresholding," IEEE Trans. Med. Imag., vol. 22, no. 9, pp. 1089-1099, Sept 2003.

[8] P. Vuylsteke, and E. Schoeters, "Multiscale Image Contrast Amplification", SPIE Image Processing, vol. 2167, pp. 551-560, 1994.
[9] K. Nambu and H. Iseki, "A noise reduction method based on a statistical test of high dimensional pixel vectors for dynamic and volumetric images," Rivista di Neuroradiologia, vol. 18, no. 1, pp. 21-33, 2005.

[10] M. Nishiki, K. Shiraishi, T. Sakaguchi, and K. Nambu, "Method for reducing noise in $\mathrm{X}$-ray images by averaging pixels based on the normalized difference with the relevant pixel," Radiological Physics and Technology, vol. 1, no. 2, pp. 188-195, Jul 2008.

[11] C. Tomasi and R. Manduchi, "Bilateral filtering for gray and color images," Sixth International Conference on Computer Vision (IEEE Cat. No.98CH36271), Jan 1998, pp. 839-846.

[12] A. Buades, B. Coll, and J. M. Morel, "A non-local algorithm for image denoising," 2005 IEEE Computer Society Conference on Computer Vision and Pattern Recognition (CVPR'05), vol. 2, pp. 60-65, June 2005.

[13] E. L. Nickoloff, "Aapm/rsna physics tutorial for residents: Physics of flat-panel fluoroscopy systems," RadioGraphics, vol. 31, no. 2, pp. 591-602, 2011.

[14] M. J. Yaffe, "Detectors for digital mammography," in Digital Mammography, U. Bick and F. Diekmann, Eds. Berlin, Heidelberg: Springer Berlin Heidelberg, 2010, pp. 13-31. [Online].

[15] Q. Yang, "Recursive Bilateral Filtering,", Computer Vision - ECCV 2012: 12th European Conference on Computer Vision, Florence, Italy, October 2012, pp. 399-413.

[16] P. Thevenaz, D. Sage, and M. Unser, "Bi-exponential edgepreserving smoother," IEEE Transactions on Image Processing, vol. 21, no. 9, pp. 3924-3936, Sept 2012.

[17] V. Ostojić, ĐordeStarčević, and V. Petrović, "Recursive anisotropic diffusion denoising," Electronics Letters, vol. 52, no. 17, pp. 1449 $1451,2016$.

[18] V. Ostojić, Đ Starčević and V. Petrović, "Recursive radiography image denoising," 2017 25rd Telecommunications Forum Telfor (TELFOR),Belgrade, 2017, pp. 1-4.

[19] P. Perona and J. Malik, "Scale-space and edge detection using anisotropic diffusion," IEEE Transactions on Pattern Analysis and Machine Intelligence, vol. 12, no. 7, pp. 629-639, Jul 1990.

[20] I. Frosio, M. Lucchese, and N. A. Borghese, "A new and reliable poisson noise estimator for radiographic images," 14th International Conference on Image Analysis and Processing (ICIAP 2007), Sept 2007, pp. 725-730.

[21] M. Makitalo and A. Foi, "Optimal inversion of the anscombe transformation in low-count poisson image denoising," IEEE Trans. Image Process., vol. 20, no. 1, pp. 99-109, Jan 2011.

[22] V. Ostojić, D. Starčević, and V. Petrović, "Artifact reduction in multiscale contrast enhancement for digital radiography," 22nd Telecommunications Forum Telfor (TELFOR), pp. 513-516, 2014.

[23] P. Gravel, G. Beaudoin and J. A. De Guise, "A method for modeling noise in medical images," in IEEE Transactions on Medical Imaging, vol. 23, no. 10, pp. 1221-1232, Oct. 2004.

[24] Z. Wang, A. Bovik, H. Sheikh and E.Simoncelli, "Image quality assessment: from error visibility to structural similarity," IEEE Trans. Image Process., vol. 13, no. 4, pp. 600-612, April 2004. 